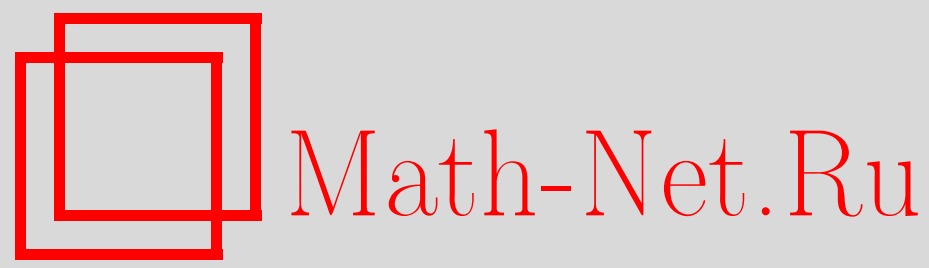

В. Т. Худалов, Аппроксимативные свойства положительной и отрицательной частей элемента в упорядоченных банаховых пространствах, $M a$ тем. заметки, 1996, том 60, выпуск 5, 793-798

DOI: https://doi.org/10.4213/mzm1896

Использование Общероссийского математического портала MathNet.Ru подразумевает, что вы прочитали и согласны с пользовательским соглашением

http://www . mathnet.ru/rus/agreement

Параметры загрузки:

IP: 3.95 .254 .165

26 апреля 2023 г., 18:36:25

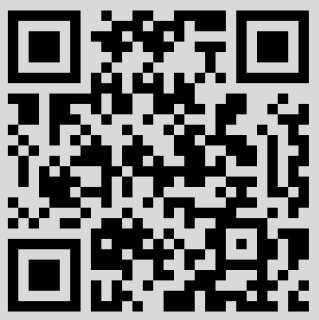




\section{АППРОКСИМАТИВНЫЕ СВОЙСТВА ПОЛОЖИТЕЛЬНОЙ И ОТРИЦАТЕЛЬНОЙ ЧАСТЕЙ ЭЛЕМЕНТА В УПОРЯДОЧЕННЫХ БАНАХОВЫХ ПРОСТРАНСТВАХ}

\section{В.Т. Худалов}

Пусть $E$ - банахово пространство над полем $\mathbb{R}$ действительных чисел, упорядоченное с помощью замкнутого конуса $E_{+}[1]$.

ОПРЕДЕЛЕНИЕ 1. Упорядоченное банахово пространство называется регулярно упорядоченным [2] (пишем $\left(E, E_{+}\right) \in(\mathscr{R})$ ), если выполнены следующие условия:

1) $\forall x, y \in E \pm x \leqslant y \Longrightarrow\|x\| \leqslant\|y\|$;

2) $\forall x \in E \quad \exists y \in E_{+}$такой, что $\pm x \leqslant y$ и $\|x\|=\|y\|$.

Для любого $x \in E$ обозначим через $|X|$ множество элементов $y \in E$ таких, что $\pm x \leqslant y$ и $\|x\|=\|y\|$. Положим $X_{+}=\frac{1}{2} x+\frac{1}{2}|X|, X_{-}=-\frac{1}{2} x+\frac{1}{2}|X|$. Множества $X_{+}$и $X_{-}$будем называть множсествами положительных (соответственно отрицательных) частей элементах.

ЗАмеч АниЕ. Нетрудно показать, что если $E$ - строго выпуклое банахово пространство [3], то множества $|X|, X_{+}, X_{-}$- одноточечные, и можно говорить о метрическом модуле, метрических положительной и отрицательной частях элемента $x$.

ОПРЕДЕЛЕНИЕ 2 . Пусть $\left(E, E_{+}\right) \in(\mathscr{R})$. Конус $E_{+}$назовем достижимым, если $\forall x \in E$ сушествует элемент $P x \in E_{+}$, на котором реализуется минимум в формуле расстояния от $x$ до $E_{+}$:

$$
d\left(x, E_{+}\right)=\inf \left\{\|a-x\|: a \in E_{+}\right\}=\|P x-x\| .
$$

Множество всех таких $P x$ обозначим через $M(x)$.

В настоящей работе исследуется задача: для каких упорядоченных банаховых пространств $M(x)=X_{+}$для любого $x \in E$. Наличие данного свойства позволяет, с одной стороны, находить расстояние от элемента до конуса, а также ближайшие для заданного элемента точки конуса $E_{+}$, а с другой стороны, дает метрическую характеристику положительной и отрицательной частей элемента. В данной статье полностью решается указанная выше задача для произвольного упорядоченного гильбертова пространства из класса $(\mathscr{R})$ и произвольного банахова пространства из класса $(\mathscr{R})$ с адлитивной на конусе нормой.

Так как гильбертово пространство строго выпукло, для любого упорядоченного гильбертова пространства $H$ с замкнутьм конусом $H_{+}$, принадлежащего классу $(\mathscr{R})$, определены для любого $x$ из $H$ его метрическая положительная и метрическая отрицательная части $x_{+}$и $x_{-}$. С другой стороны, поскольку конус $H_{+}-$замкнутое выпуклое множество в гильбертовом пространстве, $\forall x \in H$ существует проекция элемента $x$ на $H_{+}$, которую обозначим через $P x$ [4]. Следующая теорема устанавливает связь между проекцией $P x$, расстоянием элемента $x$ до $H_{+}$и элементами $x_{+}$и $x_{-}$.

ТЕОРема 1. Пусть $H \in(\mathscr{R})$ - произвольное регулярно упорядоченное гильбертово пространство с замкнутым конусом $H_{+}$. Тогда

1) $\forall x \in H \quad P x=x_{+}, d\left(x, H_{+}\right)=\left\|x_{-}\right\|$;

2) $\forall x \in H, x \notin \pm H_{+} x_{+}$u $x_{-}$принадлехсат граниче конуса $H_{+}$. 
ДоКАЗАТЕЛЬСТво. 1) Возьмем произвольный элемент $x \in H$. Если $x \in H_{+}$, то, очевидно, $P x=x, x_{+}=x, x_{-}=0$, и тогда $x=P x$ и $d\left(x, H_{+}\right)=0=\left\|x_{-}\right\|$, т.е. условие 1$)$ теоремы выполнено. Пусть теперь $x \notin H_{+}$. Тогда существует единственный вектор $P x \in H_{+}$такой, что

$$
\|P x-x\|=\inf \left\{\|a-x\|: a \in H_{+}\right\}=d\left(x, H_{+}\right) .
$$

Положим $P x-x=v$, тогда $x=P x-v$. Покажем, что $v \in H_{+}$. Действительно, если $v \notin H_{+}$, то $v=v_{+}-v_{-}$, где $v_{+}, v_{-} \in H_{+}$и $\left\|v_{+}+v_{-}\right\|=\left\|v_{+}-v_{-}\right\|$. Отсюда следует, что

$$
\left\|P x+v_{-}-x\right\|=\left\|v+v_{-}\right\|=\left\|v_{+}\right\| \geqslant\|P x-x\|=\left\|v_{+}+v_{-}\right\| .
$$

С другой стороны, $v_{+} \leqslant v_{+}+v_{-}$, отсюда $\left\|v_{+}\right\| \leqslant\left\|v_{+}+v_{-}\right\|$, что влечет $\left\|v_{+}\right\|=\left\|v_{+}+v_{-}\right\|$или $\left\|v_{+}\right\|^{2}=\left\|v_{+}\right\|^{2}+2\left(v_{+}, v_{-}\right)+\left\|v_{-}\right\|^{2}$. Посколкку равенство $\left\|v_{+}+v_{-}\right\|=\left\|v_{+}-v_{-}\right\|$равносильно $\left(v_{+}, v_{-}\right)=0$, то получаем $\left\|v_{-}\right\|^{2}=0$, т.е. $v_{-}=0$, следовательно, $v \in H_{+}$.

Докажем, что в действительности $P x=x_{+}, v=x_{-}$. Так как $x \notin H_{+}$, то $x_{-} \neq 0$. Можно считать, что $x_{+} \neq 0$, иначе $\inf \left\{\|a-x\|: a \in H_{+}\right\}=\left\|x_{-}\right\|$при $P x=0=x_{+}$. Имеем $\|P x-x\|=P x-x_{+}+x_{-} \|=\inf \left\{\|a-x\|: a \in H_{+}\right\}$. Тогда, очевидно, $P x$ доставляет минимум следующей задаче:

$$
\left\|a-x_{+}+x_{-}\right\|^{2} \rightarrow \inf ,
$$

где инфимум берется по всем $a \in H_{+}$.

Пусть $a \in H_{+}-$произвольный положительный элемент. Представим $a$ в виде $a=a_{1}+a_{2}$, где $a_{1}-$ проекция на плоскость $\operatorname{lin}\left\{x_{+}, x_{-}\right\}$, а вектор $a_{2}$ ортогонален плоскости $\operatorname{lin}\left\{x_{+}, x_{-}\right\}$, т.е. $\left(a_{2}, x_{+}\right)=0,\left(a_{2}, x_{-}\right)=0$.

Ниже будет показано, что $a_{1}=\alpha x_{+}+\beta x_{-}$, где $\alpha, \beta \geqslant 0$, т.е. $a_{1} \in H_{+}$. Имеем $\left\|a-x_{+}+x_{-}\right\|^{2}=\left\|a_{1}-x_{+}+x_{-}\right\|^{2}+\left\|a_{2}\right\|^{2}$. Отсюда следует, что инфимум в задаче (1) достигается на положительных векторах, лежащих в $\operatorname{lin}\left\{x_{+}, x_{-}\right\}$, т.е. при $a_{2}=0$. При этом

$$
\left\|a_{1}-x_{+}+x_{-}\right\|^{2}=(\alpha-1)^{2}\left\|x_{+}\right\|^{2}+(\beta+1)^{2}\left\|x_{-}\right\|^{2} .
$$

Так как

$$
H_{+} \cap \operatorname{lin}\left\{x_{+}, x_{-}\right\}=\left\{\alpha x_{+}+\beta x_{-}: \alpha, \beta \geqslant 0\right\}
$$

в силу единственности регулярно упорядочивающего конуса в гильбертовом пространстве [5], то инфимум в (2) будет при $\alpha=1, \beta=0$, т.е. если $a_{1}=x_{+}$. Следовательно, инфимум в задаче (1) достигается при $a=x_{+}$. Вследствие единственности получаем, что $P x=x_{+}$, тогда $v=x_{-}$и

$$
d\left(x, H_{+}\right)=\left\|x_{-}\right\| .
$$

Осталось показать, что $\forall a \in H_{+}$проекция $a_{1}$ на плоскость $\operatorname{lin}\left\{x_{+}, x_{-}\right\}$имеет вид: $a_{1}=\alpha x_{+}+\beta x_{-}$, где $\alpha, \beta \geqslant 0$. Действительно, пусть $a \in \operatorname{lin}\left\{x_{+}, x_{-}\right\}$, тогда в силу (3) $a=\alpha x_{+}+\beta x_{-}$, где $\alpha, \beta \geqslant 0$. Предположим, что $a \notin \operatorname{lin}\left\{x_{+}, x_{-}\right\}$. Рассмотрим трехмерное подпространство $\operatorname{lin}\left\{x_{+}, x_{-}, a\right\}$ гильбертова пространства $H$. Ясно, что

$$
H_{+} \cap \operatorname{lin}\left\{x_{+}, x_{-}, a\right\} \supset\left\{\alpha x_{+}+\beta x_{-}+\gamma a: \alpha, \beta, \gamma \geqslant 0\right\} .
$$


Предположим, что проекция $a_{1}$ вектора $a$ на плоскость $\operatorname{lin}\left\{x_{+}, x_{-}\right\}$не принадлежит множеству $\left\{\alpha x_{+}+\beta x_{-}: \alpha, \beta \geqslant 0\right\}$ и $a_{1}=\alpha x_{+}+\beta x_{-}$, где $\alpha$ или $\beta$ меньше нуля. Пусть для определенности $\alpha<0$. Рассмотрим плоскость $\operatorname{lin}\left\{x_{+}, a\right\}$. Имеем

$$
\cos \left(\widehat{x_{+}, a}\right)=\frac{\left(x_{+}, \alpha x_{+}+\beta x_{-}+a_{2}\right)}{\left\|x_{+}\right\|\|a\|}=\frac{\alpha\left\|x_{+}\right\|}{\|a\|}<0 .
$$

Так как $H_{+} \cap \operatorname{lin}\left\{x_{+}, a\right\} \supset\left\{\alpha x_{+}+\beta a: \alpha, \beta \geqslant 0\right\}$, то конус, натянутый на векторы $a$ и $x_{+}$, является регулярным в $\operatorname{lin}\left\{x_{+}, a\right\}$, так как содержит регулярный конус, натянутый на $x_{+}$и $b$, где $b=\alpha x_{+}+\beta a$ с $\alpha, \beta \geqslant 0$ и $\left(b, x_{+}\right)=0$, что противоречит единственности конуса в гильбертовом пространстве. Подобным же образом случай $\beta<0$ приводит к противоречию. Следовательно, $a_{1}=\alpha x_{+}+\beta x_{-}$, где $\alpha, \beta \geqslant 0$, и условие 1) теоремы доказано полностью.

Докажем условие 2). Пусть $x \in H, x \notin \pm H_{+}$. Предположим, что $x_{+}-$ внутренняя точка конуса $H_{+}$, т.е. существует открытый шар $B\left(x_{+}, \beta\right)$ такой, что $B\left(x_{+}, \beta\right) \subset H_{+}$. Тогда если $\rho<2 \beta$, то $x_{+}-\left(\rho x_{-}\right) /\left(2\left\|x_{-}\right\|\right) \in B\left(x_{+}, \beta\right)$, значит, $x_{+}-\left(\rho x_{-}\right) /\left(2\left\|x_{-}\right\|\right) \in H_{+}$. Отсюда при $\rho<\min \left\{2 \beta, 2\left\|x_{-}\right\|\right\}$имеем

$$
\left\|x_{+}-\frac{\rho x_{-}}{2\left\|x_{-}\right\|}-x_{+}+x_{-}\right\|=\left(1-\frac{\rho}{2\left\|x_{-}\right\|}\right)\left\|x_{-}\right\|<\left\|x_{-}\right\| .
$$

Противоречие, так как по доказанному в условии 1) имеем

$$
\left\|x_{-}\right\|=\inf \left\{\|a-x\|: a \in H_{+}\right\} .
$$

Предположим теперь, что $x_{-}-$внутренняя точка конуса $H_{+}$. Тогда найдется $\beta>0, \beta<1 / 2$ такое, что $x_{-}-\beta x_{+} \in H_{+}$. Имеем

$$
\pm\left[(1-\beta) x_{+}-\left(x_{-}-\beta x_{+}\right)\right] \leqslant(1-\beta) x_{+}+x_{-}-\beta x_{+}=(1-2 \beta) x_{+}+x_{-},
$$

откуда $\|x\|^{2} \leqslant\left\|(1-2 \beta) x_{+}+x_{-}\right\|^{2}$ или $\left\|x_{+}\right\|^{2}+\left\|x_{-}\right\|^{2} \leqslant(1-2 \beta)^{2}\left\|x_{+}\right\|^{2}+\left\|x_{-}\right\|^{2}$, противоречие. Теорема доказана полностью.

Прежде чем сформулировать следующую теорему, дадим еще одно определение.

ОПРЕДЕЛЕНИЕ 3 . Пусть $\left(E, E_{+}\right) \in(\mathscr{R})$. Достижимый конус $E_{+}$называется вполне достижимым, если $M(x)=X_{+}$для любого $x$.

Tеорема 2. Пусть $\left(E, E_{+}\right) \in(\mathscr{R})$ и норма аддитивна на конусе $E_{+}$. Тогда $E_{+}-$вполне достижимий конус.

ДоКАЗАтЕЛЬСтво. Если $x \in E_{+}$, то ясно, что $M(x)=\{x\}$. С другой стороны, если $y \geqslant \pm x$ и $y>x$, то $y-x=v \in E_{+}, v \neq 0$. В силу аддитивности нормы на конусе $\|y\|=\|x\|+\|v\|$. Поэтому если $\|y\|=\|x\|$, то $\|v\|=0$ и $y=x$, т.е. $X_{+}=\{x\}$, значит, $M(x)=X_{+}$. Возьмем произвольный $x \notin E_{+}$и докажем, что $X_{+} \subset M(x)$. Имеем $x=x_{+}-x_{-},\|x\|=\left\|x_{+}+x_{-}\right\|=\left\|x_{+}\right\|+\left\|x_{-}\right\|$, т.е. $\left\|-x_{+}+x_{-}\right\|=\left\|-x_{+}\right\|+\left\|x_{-}\right\|$, значит, норма аддитивна на элементах $-x_{+}$ и $x_{-}$. Тогда $\exists f \in E^{*},\|f\|=1$, такой, что $f\left(-x_{+}\right)=\left\|x_{+}\right\|, f\left(x_{-}\right)=\left\|x_{-}\right\|$. Так как $E \in(\mathscr{R})$ и норма аддитивна на конусе, то $E^{*} \in(\mathscr{R})$, откуда $f=g-h$, где $g, h \in E_{+}^{*}$ и $\|f\|=\|g-h\|=\|g+h\|$, значит, $\|g\| \leqslant 1,\|f\| \leqslant 1$. Имеем $-g\left(x_{+}\right)+h\left(x_{+}\right)=\left\|x_{+}\right\|, g\left(x_{-}\right)-h\left(x_{-}\right)=\left\|x_{-}\right\|$. Из второго равенства $g\left(x_{-}\right)=\left\|x_{-}\right\|+h\left(x_{-}\right)$, но $h\left(x_{-}\right) \geqslant 0$, a $g\left(x_{-}\right) \leqslant\|g\| \cdot\left\|x_{-}\right\| \leqslant\left\|x_{-}\right\|$, следовательно, $h\left(x_{-}\right)=0$ и $g\left(x_{-}\right)=\left\|x_{-}\right\|$. Первое равенство дает $h\left(x_{+}\right)=\left\|x_{+}\right\|+g\left(x_{+}\right)$, 
откуда аналогичньм образом получаем $g\left(x_{+}\right)=0, h\left(x_{+}\right)=\left\|x_{+}\right\|$. Итак, существует $g \in E_{+}^{*}$ такой, что $g\left(x_{+}\right)=0$ и $g\left(x_{-}\right)=\left\|x_{-}\right\|$, причем $\|g\|=1$. Покажем, что на $x_{+}$достигается минимум в формуле

$$
d\left(x, E_{+}\right)=\inf \left\{\|x-a\|: a \in E_{+}\right\},
$$

т.е. $d\left(x, E_{+}\right)=\left\|x-x_{+}\right\|$. Для любого $a \in E_{+}$имеем

$$
\|a-x\| \geqslant g(a)-g(x) \geqslant 0-g(x)=g\left(x_{+}\right)-g(x)=g\left(x_{-}\right)=\left\|x_{-}\right\|=\left\|x_{+}-x\right\|,
$$

т.е. $d\left(x, E_{+}\right)=\left\|x_{+}-x\right\|$, следовательно, $x_{+} \in M(x)$.

Докажем обратное включение, т.е. что $M(x) \subset X_{+}$. Пусть $P x \in M(x)$. Тогда $P x-x=v \in E_{+}$. Действительно, если $v \notin E_{+}$, то $v=v_{+}-v_{-}$, где $v_{+}, v_{-} \in E_{+}$ и $\left\|v_{+}-v_{-}\right\|=\left\|v_{+}+v_{-}\right\|=\left\|v_{+}\right\|+\left\|v_{-}\right\|$. Отсюда следует, что $P x+v_{-} \in E_{+}$ и $\left\|P x+v_{-}-x\right\|=\left\|v_{+}\right\| \geqslant\|v\|=\left\|v_{+}\right\|+\left\|v_{-}\right\|$, откуда $\left\|v_{-}\right\|=0$, т.е. $v_{-}=0$. Противоречие.

Если $x=x_{+}-x_{-}$, где $x_{+}, x_{-} \in E_{+},\left\|x_{+}-x_{-}\right\|=\left\|x_{+}+x_{-}\right\|$, то $P x+x_{-}=$ $x_{+}+v$. В силу аддитивности нормы на конусе имеем

$$
\|P x\|+\left\|x_{-}\right\|=\left\|x_{+}\right\|+\|v\|, \quad\|x\|=\left\|x_{+}\right\|+\left\|x_{-}\right\| .
$$

Но $x=P x-v$, откуда $\|x\| \leqslant\|P x\|+\|v\|$, тогда $\|P x\|+\|v\|=2\|P x\|+\left\|x_{-}\right\|-\left\|x_{+}\right\| \geqslant$ $\left\|x_{+}\right\|+\left\|x_{-}\right\|$или $\|P x\| \geqslant\left\|x_{+}\right\|$. Кроме того, $\left\|x_{-}\right\|=\left\|x_{+}-x\right\| \geqslant\|P x-x\|=\|v\|$. Поскольку $\|P x\|-\left\|x_{+}\right\|+\left\|x_{-}\right\|-\|v\|=0$, то $\|P x\|=\left\|x_{+}\right\|,\left\|x_{-}\right\|=\|v\|$, тогда $x=P x-v$ и $\|P x-v\|=\|x\|=\left\|x_{+}\right\|+\left\|x_{-}\right\|=\|P x\|+\|v\|=\|P x+v\|$, т.е. $P x \in X_{+}$. Теорема доказана полностью.

Установим теперь еще одно свойство вполне достижимого конуса.

ОПРЕДЕЛЕНИЕ 4 . Пусть $\left(E, E_{+}\right) \in(\mathscr{R})$. Конус $E_{+}$называется вполне резулярным, если $\forall x \in E$ и $\forall x_{+}, x_{-} \in E_{+}$таких, что $x=x_{+}-x_{-}$и $\left\|x_{+}-x_{-}\right\|=$ $\left\|x_{+}+x_{-}\right\|$выполняется равенство

$$
\left\|x_{+}-\lambda x_{-}\right\|=\left\|x_{+}+\lambda x_{-}\right\| \quad \forall \lambda \in \mathbb{R} .
$$

ТЕОРема 3. Всякий вполне достижимый конус вполне регулярен.

ДокАЗАТЕЛЬСтво. Возьмем произвольный $x \in E$. Так как $E_{+}-$вполне достижимый конус, то для любого $x_{+}$имеем $x_{+}=P x$. Тогда согласно предложению 0.3 в [4] если $z$ принадлежит линейному отрезку с концами $x$ и $x_{+}$, то $P z=x_{+}$. Поэтому для элемента

$$
(1-\lambda) x_{+}+\lambda x=\lambda\left(x_{+}-x_{-}\right)+(1-\lambda) x_{+}=x_{+}-\lambda x_{-}
$$

имеем $P\left(x_{+}-\lambda x_{-}\right)=P x=x_{+}$, значит, найдется такая положительная часть элемента $x_{+}-\lambda x_{-}$, что $\left(x_{+}-\lambda x_{-}\right)_{+}=x_{+}$, отсюда $\left(x_{+}-\lambda x_{-}\right)_{-}=\lambda x_{-}$. И так как для любых элементов $x_{+}, x_{-}$имеет место равенство $\left\|x_{+}-x_{-}\right\|=\left\|x_{+}+x_{-}\right\|$, получаем

$$
\left\|x_{+}-\lambda x_{-}\right\|=\left\|x_{+}+\lambda x_{-}\right\| \quad \forall \lambda \in[0,1] .
$$

С другой стороны, $-x=x_{-}-x_{+}$, и поскольку $\|-x\|=\left\|x_{-}-x_{+}\right\|=\left\|x_{-}+x_{+}\right\|$, то в качестве $(-x)_{+}$и $(-x)$ - можно взять $x_{-}$и $x_{+}$соответственно, т.е. $\left\|x_{-}-\lambda x_{+}\right\|=$ $\left\|x_{-}+\lambda x_{+}\right\| \forall \lambda \in[0,1]$ или $\left\|(1 / \lambda) x_{-}+x_{+}\right\|=\left\|(1 / \lambda) x_{-}-x_{+}\right\| \forall \lambda \in(0,1]$. Положив $\mu=1 / \lambda$, получим $\left\|x_{+}+\mu x_{-}\right\|=\left\|x_{+}-\mu x_{-}\right\| \forall \mu \in[1,+\infty)$. Итак, $\forall \lambda \in[0,+\infty)\left\|x_{+}-\lambda x_{-}\right\|=\left\|x_{+}+\lambda x_{-}\right\|$, т.е. конус в $E_{+}$является вполне регулярным.

Из теорем 1 и 2 выводим следуюшее 
СлЕДСтвИЕ. Пусть $\left(E, E_{+}\right) \in(\mathscr{R})$ и норма аддитивна на конусе. Тогда

1) конус $E_{+}$является вполне регулярным;

2) для любого $x \in E$ нормы элементов из $X_{-}$равны мехсду собой $u$ равны $d\left(x, E_{+}\right)$;

3) для любого $x \in E$ нормы элементов из $X_{+}$равны мехсду собой $u$ равньц $\|x\|-d\left(x, E_{+}\right)$.

В заключение приведем два примера. В примере 1 показано, что даже в трехмерном пространстве $E$ из класса $(\mathscr{R})$ множества $M(x)$ и $X_{+}$, вообще говоря, не сравнимы в смысле упорядочения по теоретико-множественному включению. Пример 2 является иллюстрацией к теоремам 2 и 3.

ПримеР 1. Пусть $E=l_{1}^{3}$ и $E_{+}=\left\{x=\left(x_{1}, x_{2}, x_{3}\right): x_{1} \geqslant\left|x_{2}\right|+\left|x_{3}\right|\right\}$. Можно показать, что $\left(E, E_{+}\right) \in(\mathscr{R})$. Возьмем $x=(1,1,1)$. Нетрудно установить, что для этого элемента

$$
\begin{aligned}
& X_{+}=\left\{\left(2-\frac{\lambda}{2}, \frac{\alpha \lambda+1}{2}, \frac{(1-\alpha) \lambda+1}{2}\right): 0 \leqslant \lambda \leqslant 1,0 \leqslant \alpha \leqslant 1\right\} \\
& X_{-}=\left\{\left(1-\frac{\lambda}{2}, \frac{\alpha \lambda-1}{2}, \frac{(1-\alpha) \lambda-1}{2}\right): 0 \leqslant \lambda \leqslant 1,0 \leqslant \alpha \leqslant 1\right\} .
\end{aligned}
$$

Расстояние от $x$ до $E_{+}$равно 1 , и те из $x_{+} \in X_{+}$, для которых норма ассоциированного элемента $x_{-}$равна 1, принадлежит $M(x)$. Но $\left\|x_{-}\right\|=1$ только если $\lambda=1$. Значит,

$$
M(x) \cap X_{+}=\left\{\left(\frac{3}{2}, \frac{\alpha+1}{2}, \frac{2-\alpha}{2}\right): 0 \leqslant \alpha \leqslant 1\right\} .
$$

Непосредственное вычисление показьвает, что

$$
M(x)=\{(1+\lambda, \alpha, 1+\lambda-\alpha): 0 \leqslant \lambda \leqslant 1, \lambda \leqslant \alpha \leqslant 1\} .
$$

Для элемента $P x=(4 / 3,2 / 3,3 / 4)$, принадлежащего $M(x)$, имеем $P(x) \notin X_{+}$, т.е. множества $M(x)$ и $X_{+}$не сравнимы. Интересно отметить, что найдутся единственные ассоциированные элементы $x_{+}=(3 / 2,3 / 4,3 / 4), x_{-}=(1 / 2,-1 / 4,-1 / 4)$ такие, что для любого $t \geqslant 0$

$$
\left\|x_{+}+t x_{-}\right\|=\left\|x_{+}-t x_{-}\right\|= \begin{cases}t, & \text { если } t \geqslant 3, \\ 3, & \text { если } t<3 .\end{cases}
$$

ПримеР 2. Пусть $E=c_{0}$ над $\mathbb{R}$ с естественной нормой и конусом $E_{+}=\left\{x \in c_{0}:\right.$ $\left.x_{1} \geqslant \sup \left\{\left|x_{n}\right|: n \geqslant 2\right\}\right\}$, где $x=\left(x_{1}, \ldots, x_{n}, \ldots\right)$. Покажем, что $\left(E, E_{+}\right) \in(\mathscr{R})$.

1. Пусть $\pm x \leqslant y$. Имеем $y_{1}-x_{1} \geqslant \sup \left\{\left|y_{n}-x_{n}\right|: n \geqslant 2\right\}, y_{1}+x_{1} \geqslant$ $\sup \left\{\left|y_{n}+x_{n}\right|: n \geqslant 2\right\}$. Если $z \in E_{+}$, то $\|z\|=z_{1}$, поэтому норма аддитивна на конусе и $\|y\|=y_{1},\|y-x\|=y_{1}-x_{1},\|y+x\|=y_{1}+x_{1}$. Отсюда $2\|x\| \leqslant$ $\|y-x\|+\|y+x\|=y_{1}-x_{1}+y_{1}+x_{1}=2\|y\|$, т.е. $\|x\| \leqslant\|y\|$.

2. Пусть $x=\left(x_{1}, x_{2}, \ldots, x_{n}, \ldots\right)$ и $\left|x_{1}\right| \geqslant \sup \left\{\left|x_{n}\right|: n \geqslant 2\right\}$. Тогда если $x_{1}>0$, то можно взять $y=x$; если $x_{1}<0$, то $y=-x$. Легко видеть, что если $x \in E_{+}$, то $X_{+}=\{x\}, X_{-}=\{0\}$; если же $x \in-E_{+}$, то $X_{+}=\{0\}, X_{-}=\{x\}$. 
Предположим теперь, что $\left|x_{1}\right|<\sup \left\{\left|x_{n}\right|: n \geqslant 2\right\}$. Положим $\sup \left\{\left|x_{n}\right|:\right.$ $n \geqslant 2\}=a$. Возьмем

$$
y=\left(a, \frac{x_{1} x_{2}}{a}, \ldots, \frac{x_{1} x_{n}}{a}, \ldots\right), \quad y \in c_{0}
$$

Тогда $y \pm x \in E_{+}$и $\|y\|=\sup \left\{a,\left|x_{1} x_{n}\right| / a: n \geqslant 2\right\}=\sup \left\{a,\left|x_{1}\right|\right\}=\|x\|$. Обозначим $Y=\left\{y \in E_{+}: y \geqslant \pm x,\|y\|=\|x\|\right\}$. Пусть $y=\left(y_{1}, \ldots, y_{n}, \ldots\right) \geqslant$ $\pm\left(x_{1}, \ldots, x_{n}\right),\|y\|=\|x\|$. Тогда $y=\left(a, y_{2}, \ldots, y_{n}, \ldots\right)$, где

$\max \left\{-a-x_{1}-x_{i},-a+x_{1}+x_{i}\right\} \leqslant y_{i} \leqslant \min \left\{a+x_{1}-x_{i}, a-x_{1}+x_{i}\right\}, \quad i=2, \ldots$

В этом случае $X_{+}=\frac{1}{2} x+\frac{1}{2} Y ; X_{-}=\frac{1}{2} Y-\frac{1}{2} x$. Все элементы из $X_{+}$имеют норму $\frac{1}{2}\left(a+x_{1}\right)$, авсе элементы из $X_{-}-$норму $\frac{1}{2}\left(a-x_{1}\right)$, кроме того, $d\left(x, E_{+}\right)=\frac{1}{2}\left(a-x_{1}\right)$. Если, например, $x=(1,2,4,0, \ldots, 0, \ldots)$, то $Y=\left\{\left(4, y_{2}, y_{3}, \ldots, y_{n}, \ldots\right)\right.$ : $\left.\lim y_{n}=0\right\}$, где $-1 \leqslant y_{2} \leqslant 3, y_{3}=1,-3 \leqslant y_{n} \leqslant 3, n=4,5, \ldots$ Здесь

$$
\begin{aligned}
& X_{+}=\left\{\left(\frac{5}{2}, 1+z_{2}, \frac{5}{2}, z_{4}, \ldots, z_{n}\right):\right. \\
& \left.\frac{1}{2} \leqslant z_{2} \leqslant \frac{3}{3},\left|z_{n}\right| \leqslant \frac{3}{2}, n=4, \ldots, \lim _{n \rightarrow \infty} z_{n}=0\right\}, \\
& X_{-}=\left\{\left(\frac{3}{2}, u_{2}-1,-\frac{3}{2}, u_{4}, \ldots, u_{n}, \ldots\right):\right. \\
& \left.\frac{1}{2} \leqslant u_{2} \leqslant \frac{3}{2},\left|u_{n}\right| \leqslant \frac{3}{2}, n=4, \ldots, \lim _{n \rightarrow \infty} u_{n}=0\right\}
\end{aligned}
$$

и $d\left(x, E_{+}\right)=\frac{3}{2}$. Отметим также, что $\forall x_{+} \in X_{+}$и $x_{-}=x_{+}-x$ имеем

$$
\frac{5+3 t}{2}=\left\|x_{+}+t x_{-}\right\|=\left\|x_{+}-t x_{-}\right\| \quad \forall t \in \mathbb{R}_{+} .
$$

Северо-Осетинский государственный университет

Поступило 20.06.96

\section{СПИСОК ЦИТИРОВАННОЙ ЛИТЕРАТУРЫ}

1. Вулих Б.З. Введение в теорию конусов в нормированных пространствах. Калинин: Изд-во Калининского ун-та, 1977. 2. Худалов В. Т. // Сиб. матем. ж. 1996. Т. 1. № 1. С. 193-196. 3. Дистель Д. Геометрия банаховых пространств. Киев: Вища школа, 1980. 4. Власов Л. П. // УМН. 1973. Т. 28. №6. С. 3-66. 5. Худалов В.Т. Тензорные произведения банаховых пространств с конусами. Дисс. ... к.ф.-м.н. Л., 1980. 